\title{
THE STABLE SOLUTIONS OF QUADRATIC MATRIX EQUATIONS
}

\author{
STEPHEN CAMPBELL AND JOHN DAUGHTRY
}

\begin{abstract}
The authors determine which solutions $K$ to the quadratic matrix equation $X B X+X A-D X-C=0$ are "stable" in the sense that all small changes in the coefficients of the equation produce equations some of whose solutions are close to $K$ (in the metric determined by the operator norm). Our main result is that a solution is stable if and only if it is an isolated solution. (The isolated solutions already have a simple characterization in terms of the coefficient matrices.) It follows that each equation has only finitely many stable solutions.

Equivalently, we identify the stable invariant subspaces for an operator $T$ on a finite-dimensional space as the isolated invariant subspaces.
\end{abstract}

Let $\mathbf{H}$ and $\mathbf{K}$ be finite-dimensional complex inner product spaces with $A$ in $\mathbf{B}(\mathbf{H}), D$ in $\mathbf{B}(\mathbf{K}), B$ in $\mathbf{B}(\mathbf{K}, \mathbf{H})$, and $C$ in $\mathbf{B}(\mathbf{H}, \mathbf{K})$, where $\mathbf{B}()$ denotes a set of bounded linear transformations in the usual manner. We study the operator equation

$$
F(X)=X B X+X A-D X-C=0
$$

for $X$ in $\mathbf{B}(\mathbf{H}, \mathbf{K})$. The study of (1) is important for many applications, including the quadratic eigenvalue problem and aspects of the theory of optimal control. (See [5], [1], and the bibliographies there.)

In general, solutions to (1) may fluctuate wildly when the coefficients are changed slightly. Indeed, the equation $X A-A X=0$ (for any square matrix $A$ ) has many solutions while $X A-A X=\varepsilon I$ has none (where $\varepsilon$ is any nonzero complex number and $I$ is the identity matrix of appropriate dimension). Obviously, this possibility is devastating when the choice of coefficient matrices may involve small errors.

It is well known that if $K$ is a solution to (1) such that $F^{\prime}(K)$ (the Fréchet derivative of $F$ at $K$ ) is invertible, then $K$ is a "stable" solution in the following sense: for any $\varepsilon>0$, making each coefficient of the perturbed equation $X \mathscr{B} X+X \mathscr{Q}-\mathscr{D} X-\mathcal{C}=0$ sufficiently close (in operator norm) to the corresponding coefficient of (1) ensures that the new equation has a solution $\mathscr{K}$ with $\|\mathscr{K}-K\|<\varepsilon$. (\|・\| denotes the operator norm.) Of course, the invertibility of $F^{\prime}(K)$ is equivalent to the disjointness of the spectra of $A+B K$ and $D-K B[8]$. Our necessary and sufficient condition for stability is closely related to this criterion:

Received by the editors March 28, 1978.

AMS (MOS) subject classifications (1970). Primary 15A24, 47A55.

Key words and phrases. Perturbation theory, stable invariant subspaces, quadratic matrix equations. 
THEOREM. The following properties of a solution $K$ to (1) are equivalent:

(i) $K$ is a stable solution.

(ii) $O$ is a stable solution to $X B X+X(A+B K)-(D-K B) X=0$.

(iii) The common eigenvalues for $A+B K$ and $D-K B$, considered as eigenvalues of

$$
T=\left(\begin{array}{cc}
A+B K & B \\
0 & D-K B
\end{array}\right),
$$

acting on $\mathbf{H} \oplus \mathbf{K}$, have geometric multiplicity one.

(iv) $K$ is an isolated point of the solution set.

(v) Every noncyclic generalized eigenspace $\mathbf{M}$ for $T$ (defined in (iii)) satisfies $\mathbf{M} \cap \mathbf{H}=\{0\}$ or $\mathbf{M}$.

(vi) The intersection of the graph of $K$ with each noncyclic generalized eigenspace $\mathbf{M}$ for $S=\left(\begin{array}{ll}A & B \\ C & D\end{array}\right)$ is either $\{0\}$ or $\mathbf{M}$.

REMARK. It follows from (vi) that (1) can have only finitely many stable solutions.

The equivalence of (iii)-(vi) was proved in [2] and will be assumed here. (The equivalence of (iv) and (vi) is really a translation to a different setting of a result from [4].) What we shall prove here is the equivalence of (i) and (vi) and the equivalence of (ii) and (v). We will need the homeomorphism $g$ between solutions to (1) and certain invariant subspaces for $S$. Define the distance between two subspaces of $\mathbf{H} \oplus \mathbf{K}$ to be the (operator) distance between their orthogonal projections. Then $g$ takes $K$ (a solution to (1)) to the graph of $K$. The range of $g$ is the set of invariant subspaces for $S$ whose distance from $\mathbf{H}$ is less than one [2, Theorem 1]. We call an invariant subspace $\mathbf{M}$ for an operator $S$ "stable" if given $\varepsilon>0$ there exists $\delta>0$ such that $\|S-L\|<\delta$ for some operator $L$ implies that $L$ has an invariant subspace $\mathbf{M}^{\prime}$ with $\operatorname{dist}\left(\mathbf{M}, \mathbf{M}^{\prime}\right)<\varepsilon$.

In order to establish the desired equivalences, it suffices to prove:

THEOREM. An invariant subspace for an operator $S$ on a finite-dimensional Hilbert space $\mathbf{L}$ is stable if and only if it is an isolated point of the set of invariant subspaces for $S$.

Proof. Suppose $\mathbf{M}$ is an isolated invariant subspace for $S$, but there exist an $\varepsilon>0$ and a sequence $S_{m}$ of operators converging to $S$ such that $\operatorname{dist}\left(\mathbf{N}_{m}, \mathbf{M}\right)>\varepsilon$ for each choice of $\mathbf{N}_{m}$, an invariant subspace for $S_{m}$. Let $\mathbf{L}=+_{i=1}^{n} \mathbf{M}_{i}$ be the primary decomposition for $S$. (Each $\mathbf{M}_{i}$ is the span of all the generalized eigenvectors that go with a particular eigenvalue of $S$.) The primary summands for $S_{m}$ may be partitioned so that if $\mathbf{L}_{i, m}$ is the span of one equivalence class of summands, then $\mathbf{L}_{i, m}$ converges to $\mathbf{M}_{i}$ as $m$ approaches infinity [7, p. 106]. There exists some index (which we call 1 ) such that $\mathbf{M} \cap \mathbf{M}_{1}$ is not the limit of any sequence $\mathbf{Q}_{m}$ of subspaces of $\mathbf{L}_{1, m}$ with $\mathbf{Q}_{m}$ invariant for $S_{m}$. We may choose a sequence $\mathbf{Q}_{m} \subset \mathbf{L}_{I, m}$ of invariant 
subspaces for $S_{m}$ such that

$$
\operatorname{dim} \mathbf{Q}_{m}=\operatorname{dim}\left(\mathbf{M} \cap \mathbf{M}_{1}\right)
$$

and $\mathbf{Q}_{m}$ converges to $\mathbf{Q} \subset \mathbf{M}$. $\mathbf{Q}$ is then an invariant subspace for $S$. However, $\mathbf{M}_{1}$ is cyclic by the Douglas-Pearcy characterization of isolation (vi). Therefore, $\mathbf{M} \cap \mathbf{M}_{1}$ is the unique invariant subspace for the restriction of $S$ to $\mathbf{M}_{1}$ having its particular dimension. Consequently, $\mathbf{Q}=\mathbf{M} \cap \mathbf{M}_{1}$, which gives us a contradiction. Thus isolated solutions are stable.

Now suppose that $\mathbf{M}$ is a nonisolated invariant subspace for $S$. Suppose that $\mathbf{M}_{1}$ is one of the noncyclic primary summands for $S$ such that $\mathbf{M} \cap \mathbf{M}_{1}$ is a proper subspace of $\mathbf{M}_{1}$. Let $S_{1}$ be the restriction of $S$ to $\mathbf{M}_{1}$. We shall demonstrate that $\mathbf{M} \cap \mathbf{M}_{1}$ is unstable for $S_{1}$. Let $\mathbf{B}$ be a basis for $\mathbf{M}_{1}$ with respect to which $S_{1}$ is in Jordan form (with the off-diagonal ones below the main diagonal). $\mathbf{B}=\cup \mathbf{B}_{j}$ where each $\mathbf{B}_{j}$ is a basis for one of the cyclic summands in $\mathbf{M}_{1}$ (i.e., each $\mathbf{B}_{j}$ corresponds to one Jordan cell). We claim that the dimension of the intersection of $\mathbf{M}$ with the span of $\mathbf{B}_{j}$ is equal to the number of elements in $\mathbf{M} \cap \mathbf{B}_{j}$ for all $j$. To see this, let $\mathbf{M}_{1, j}$ denote the span of $\mathbf{B}_{j}$. We may assume that $\mathbf{M} \cap \mathbf{M}_{1}$ is stable, since otherwise we have finished this part of the proof. Choose distinct complex numbers $\varepsilon_{j}$ near 0 and define $T$ on $\mathbf{M}_{1}$ by

$$
T x=S_{I} x+\varepsilon_{j} x
$$

for $x$ in $\mathbf{M}_{1, j}$. Then each invariant subspace for $T$ splits into a direct sum of its intersections with the subspaces $\mathbf{M}_{1, j}$, and the matrix of $T$ with respect to $\mathbf{B}_{j}$ is a Jordan cell. By revising our choice of the numbers $\varepsilon_{j}$, we may take $T$ arbitrarily close to $S_{1}$. By the stability of $\mathbf{M}, \mathbf{M}$ is a limit of some sequence of invariant subspaces for the operators $T$ obtained by varying the numbers $\varepsilon_{j}$, but the invariant subspaces of $T$ do not change with the choice of these numbers, and each invariant subspace for $T$ satisfies the condition desired for M. Therefore $\mathbf{M}$ also splits.

In particular, there is some $j$ such that $\mathbf{B}_{j} \cap \mathbf{M}$ contains a nonzero vector $y$. Renumbering the sets $\mathbf{B}_{j}$ if necessary, $y$ belongs to $\mathbf{B}_{1} \cap \mathbf{M}$. Choose some nonzero number $\varepsilon$, and consider the operator $W_{\varepsilon}$ on $\mathbf{M}_{1}$ obtained from $S_{1}$ by replacing each zero entry below the main diagonal in the matrix for $S_{1}$ with $\varepsilon$. The vectors $W_{\varepsilon}^{k} y(k \geqslant 1)$ span a space including the span of $\mathbf{B}_{j}(j>1)$. Because $W_{\varepsilon}$ approaches $S_{1}$ as $\varepsilon$ approaches 0 and $\mathbf{M} \cap \mathbf{M}_{1}$ is stable, we may conclude that the distance from $y$ to some invariant subspace $\mathbf{M}_{\varepsilon}$ for $W_{\varepsilon}$ approaches 0 . Yet if $\mathbf{B}=\left\{y_{1}, y_{2}, \ldots, y_{n}\right\}$, any invariant subspace for $W_{\varepsilon}$ is simply the span of a subset of $\mathbf{B}$ having the form $\left\{y_{i}, y_{i+1}, \ldots, y_{n}\right\}$. It follows that $y$ belongs to $\mathbf{M}_{\varepsilon}$ for sufficiently small $\varepsilon$, where $\left\{\mathbf{M}_{\varepsilon}\right\}$ is a collection of subspaces approaching $\mathbf{M} \cap \mathbf{M}_{1}$. Thus the span of $\mathbf{B}_{j}$ is contained in $\mathbf{M}_{\varepsilon}$ for $j>1$ and sufficiently small $\varepsilon$. It follows that $\mathbf{B}_{j} \subset \mathbf{M} \cap \mathbf{M}_{1}$ for $j>1$. $(j$ assumes the value 2 because $\mathbf{M}_{i}$ is not cyclic for $S_{1}$.) Knowing that $\mathbf{B}_{2} \subset \mathbf{M} \cap$ $\mathbf{M}_{1}$, we may reproduce the preceding argument with $\mathbf{B}_{1}$ and $\mathbf{B}_{2}$ interchanged, 
and we may take $y=y_{1}$, the first element of $\mathbf{B}$. This time we conclude that all of $\mathbf{B}$ is contained in $\mathbf{M}$, contrary to the fact that $\mathbf{M} \cap \mathbf{M}_{1}$ was a proper subspace of $\mathbf{M}_{1}$. This error must be the result of the supposition that $\mathbf{M} \cap \mathbf{M}_{1}$ was stable for $S_{1}$, so we conclude that $\mathbf{M} \cap \mathbf{M}_{1}$ is unstable for $S_{1}$.

To prove that $\mathbf{M}$ is unstable for $S$, define $S_{\varepsilon}$ by $S_{\varepsilon}=S$ on $\mathbf{M}_{k}$ for $k \neq 1$ and choose the restriction of $S_{\varepsilon}$ to $\mathbf{M}_{1}$ so that $S_{\varepsilon} \rightarrow S_{1}$ as $\varepsilon \rightarrow 0$ and the invariant subspaces of $S_{\varepsilon}$ in $\mathbf{M}_{1}$ are bounded away from $\mathbf{M} \cap \mathbf{M}_{1}$. Every invariant subspace $\mathbf{M}_{\varepsilon}$ for $S_{\varepsilon}$ splits $\mathbf{M}_{\varepsilon}=+_{i}\left(\mathbf{M}_{\varepsilon} \cap \mathbf{M}_{i}\right)$, so the fact that $\mathbf{M}_{\varepsilon} \cap \mathbf{M}_{1}$ is bounded away from $\mathbf{M} \cap \mathbf{M}_{1}$ implies that $\mathbf{M}_{\varepsilon}$ is bounded away from $\mathbf{M}$. This proves that $\mathbf{M}$ is unstable for $S$, completing the proof of the theorem.

The argument that isolation implies stability given in the preceding theorem is easily modified to show that if $S$ is a linear operator on a finite-dimensional space and $S^{\prime}$ is sufficiently near $S$, then every connected component $\mathbf{C}^{\prime}$ of invariant subspaces for $S^{\prime}$ is close to some connected component $\mathbf{C}$ of invariant subspaces for $S$ in the sense that

$$
\sup _{\mathbf{M}^{\prime} \in \mathbf{C}^{\prime}} \inf _{\mathbf{M} \in \mathbf{C}} \operatorname{dist}\left(\mathbf{M}^{\prime}, \mathbf{M}\right)
$$

goes to 0 as $S^{\prime}$ approaches $S$.

We begin the proof with the characterization of the connected components of invariant subspaces for $S$ obtained by Douglas and Pearcy in [4]: two invariant subspaces are in the same component if and only if their intersections with each generalized eigenspace for $S$ have the same dimension. Now suppose that there exist a sequence $\left\{S_{m}\right\}$ of operators converging to $S$ and a sequence $\left\{\mathbf{C}_{m}\right\}$ with each $\mathbf{C}_{m}$ a connected component of invariant subspaces for $S_{m}$ satisfying

$$
\sup _{\mathbf{M}_{m} \in \mathbf{C}_{m}} \inf _{\mathbf{M} \in \mathbf{C}} \operatorname{dist}\left(\mathbf{M}_{m}, \mathbf{M}\right) \geqslant \delta>0
$$

for every component $\mathbf{C}$ of invariant subspaces for $S$. Define $\mathbf{L}_{i, m}$ (as in the proof of the preceding theorem) to be the span of certain primary summands for $S_{m}$ so that $\mathbf{L}_{i, m} \rightarrow \mathbf{M}_{i}$ where ${ }_{i} \mathbf{M}_{i}$ is the primary decomposition for $S$. By the same arguments as before we may assume that for any $\mathbf{C}$ as above there exists $\mathbf{M}_{m}$ in $\mathbf{C}_{m}$ such that $\mathbf{M}_{m} \cap \mathbf{L}_{1, m}$ does not approach $\mathbf{M} \cap \mathbf{M}_{1}$ for any $\mathbf{M}$ in $\mathbf{C}$, yet $\mathbf{M}_{m} \cap \mathbf{L}_{1, m}$ approaches some invariant subspace $\mathbf{N}$ for the restriction of $S$ to $\mathbf{M}_{1}$. The dimension of $\mathbf{M}_{m} \cap \mathbf{L}_{1, m}$ does not change with the choice of $\mathbf{M}_{m}$ in $\mathbf{C}_{m}$. Because all of the invariant subspaces for $S$ in $\mathbf{M}_{1}$ with the same dimension as $\mathbf{N}$ are in the same connected component as $\mathbf{N}$, we have a contradiction.

Finally, the fact that isolated invariant subspaces are stable can be extended to algebraic operators on a Hilbert space of any dimension. This is a direct consequence of the characterization of the isolated invariant subspaces of an algebraic operator given in [3], the semicontinuity of separated parts of 
the spectrum [7, p. 212], and the preceding theorem applied to the (necessarily finite-dimensional) cyclic generalized eigenspaces.

\section{BIBLIOGRAPHY}

1. W. A. Coppel, Matrix quadratic equations, Bull. Austral. Math. Soc. 10 (1974), 377-401.

2. J. P. Daughtry, Isolated solutions of quadratic matrix equations, J. Linear Algebra Appl. 21 (1978), 89-94.

3. The inaccessible invariant subspaces of certain $C_{0}$ operators (preprint).

4. R. G. Douglas and C. Pearcy, On a topology for invariant subspaces, J. Functional Analysis 2 (1968), 323-341.

5. J. Eisenfeld, Operator equations and nonlinear eigenparameter problems, J. Functional Analysis 12 (1973), 475-490.

6. K. Hoffman and R. Kunze, Linear algebra, 2nd ed., Prentice-Hall, Englewood Cliffs, N. J., 1971.

7. T. Kato, Perturbation theory for linear operators, Springer-Verlag, New York, 1966.

8. M. Rosenblum, On the operator equation $B X-X A=Q$, Duke Math. J. 23 (1956), 263-270.

Department of Mathematics, North Carolina State University, Raleigh, North Carolina 27650

Department of Mathematics, Sweet Briar College, Sweet Briar, Virginia 24595 